Die folgenden zwei Beiträge befassen sich mit hochaktuellen, in der Ärzteschaft aber bisher nicht breitdiskutierten Fragen der genetischen Labordiagnostik bzw. sogenannter «Gentests». Wir danken insbesondere Prof. Hansjakob Müller, dass er dem Wunsch der Redaktion entsprochen hat, aus der Sicht des Experten zentrale Aspekte des Themas in einem Übersichtsartikel zusammenzufassen.

Die Redaktion

\title{
Genetische Labordiagnostik - heute und morgen?
}

Hansjakob Müller

Korrespondenz:

Prof. Dr. med. Hansjakob Müller Medizinische Genetik USB

Burgfelderstrasse 101 (Haus J)

CH-4055 Basel

Tel. 0612653620

hansjakob.mueller[at]unibas.ch
Wir stehen an der Schwelle in ein neues Zeitalter der medizinisch-genetischen Diagnostik. Mit der herkömmlichen molekulargenetischen Analytik (= first generation sequencing FGS) untersuchte man nur ein einzelnes Gen, um Mutationen zu identifizieren , die zu einer bekannten monogenen Erbkrankheit (Zystische Fibrose, Huntington-Erkrankung, hereditäres Mamma-/Ovarialkarzinom und andere) prädisponieren. Heute analysiert man immer grösser werdende Anteile des gesamten Genoms und sucht dort nach Veränderungen, die sich nachteilig auf die Gesundheit auswirken könnten. Neue Verfahren ermöglichen die Generierung einer Vielzahl von DNASequenzierdaten, und dies erst noch zu einem vergleichbar günstigen Preis sowie innerhalb recht kurzer Zeit. Mit dem sogenannten Whole genome sequencing WGS (Kasten) will man die gesamte DNASequenz eines einzelnen Menschen erfassen und evaluieren. Wegen technologischen Entwicklungen in der DNA-Laboranalytik und wegen Fortschritten in der Bioinformatik wird dies immer möglicher.

Da das WGS zu klinisch nutzbaren Ergebnissen in der Differentialdiagnostik und der massgeschneiderten Therapie (personalisierte Medizin) führt, finden deren Verfahren - vorerst allerdings noch von Budget- und anderen Managementfragen (z. B. Umfang des zu erwartenden Probeneingangs) belastet unweigerlich Einzug in die Laboratorien unserer Universitäten und Spitäler sowie in Privatlabors. Bereits heute werden früh im Diagnoseprozess sogenannte Multigene panels eingesetzt, um den Ursachen von neurodegenerativen Erkrankungen, geistigen Behinderungen, nicht-syndromischen Seh- und Hörstörungen, Kardiomyopathien, familiär auftretenden Tumorkrankheiten und anderen Krankheiten/Behinderungen auf die Spur zu kommen.

Die aufgezeigte Entwicklung der DNA-Analytik wird keineswegs nur durch die akademische Forschung beeinflusst, sondern besonders auch von Industrien gesteuert, die genetische Diagnostikgeräte/ -verfahren entwickeln und verkaufen wollen, sowie von Privatlabors, die über Internet genetische Analysen anbieten (Direct-to-consumer DTC genetic indus- tries) oder die in der Schweiz mit Gentests ins Geschäft kommen wollen.

Die Antworten zu vielen mit dieser Entwicklung verbundenen Fragen sind noch offen. Vielleicht wurden nicht einmal alle diesbezüglichen Probleme schon klar erkannt. Man klebt zu sehr noch an den vom First generation sequencing stammenden Vorstellungen. Dabei ist es an der Zeit, gute Voraussetzungen für einen sinnvolle Veranlassung und vertrauenswürdige Durchführung von Whole genome sequencingAnalysen sowie einen verantwortungsvollen Umgang mit der Fülle der daraus hervorgehenden genetischen Daten zu schaffen. Eine ausgewogene Diskussion zu diesem Thema in der SÄZ ist daher wünschenswert. Auch eine Aufklärung der breiten Öffentlichkeit ist angezeigt. Die Wahrnehmung von Genetik in der Medizin muss vielerorts vorerst noch etwas «normalisiert» werden: «Genetik» bedeutet keineswegs gleich Gefahr/Bedrohung!

$\mathrm{Zu}$ den Problemkreisen, die mit dem WGS auf uns zukommen, gehören unter anderem folgende Aspekte:

- Die Schere zwischen der Produzierbarkeit von DNA-Daten einerseits und deren Translation für die Bedürfnisse des klinischen Alltags andererseits klafft vorerst immer weiter auseinander. Die Interpretation molekulargenetischer Daten erfordert die Berücksichtigung der klinischen Aus-

\section{"Whole genome sequencing» (WGS)}

Unter «Whole genome sequencing" (WGS) (Synonyme: full, complete oder entire genome sequencing) versteht man die Analyse der gesamten DNA-Sequenz des Erbgutes (Genom) eines Organismus in einem bestimmten Zeitpunkt. Das Genom umfasst die Protein-kodierenden Gene, alle weiteren funktionellen (RNAonly-Gene und andere) sowie die übrigen DNA-Sequenzen im Zellkern und in den Mitochondrien. Auch epigenetische DNA-Modifikationen werden dabei erfasst. In einer Übergangsphase sprach man vorerst vom «Next-generation sequencing» NGS). 
sagekraft und Reichweite der Befunde sowie den Umgang mit Wahrscheinlichkeiten. Ausser mit derjenigen für bestimmte Eiweiss-kodierende Gene haben wir diesbezüglich wenig Erfahrungen. Die DNA-Sequenzierung kann das Vorliegen von möglichen gesundheitlichen Risiken aufzeigen, was aber nicht heisst, dass sich diese klinisch manifestieren müssen. Zudem ist offen, ob überhaupt und wie weit verschiedene identifizierbare DNA-Risikovarianten bei einem einzelnen Individuum untereinander, respektive auch mit erworbenen Gesundheitsrisikofaktoren (Lebensstil usw.) interagieren. Um solche Wissenslücken zu schliessen, müssen vorerst Studien mit sehr vielen Teilnehmern realisiert werden können, die zur Freigabe ihrer genomischen und klinischen Daten bereit sind. Dies sollte beim gesetzlichen Regulieren des WGS berücksichtigt werden.

- Es wird nicht einfach sein, für den medizinischen Alltag allgemeingültige Massstäbe für die individuelle Interpretation von WGS-Daten aufzustellen, die besagen, ob die erhobenen überhaupt und - falls ja - in welchem Ausmass klinisch relevant sind, oder gar Computerprogramme für diesen Zweck zu entwickeln. Daher

\section{Die meisten mittels «Whole genome sequencing»} generierten Daten lassen sich klinisch nie nutzen.

ist grosse Zurückhaltung im Hinblick auf ein pränatales Whole genome sequencing (WGS) oder ein WGS bei Kindern und Jugendlichen angezeigt. Zu gross ist die Gefahr, dass bei den künftigen Eltern durch WGS-Daten unnötige Ängste ausgelöst werden, die zum Schwangerschaftsabbruch führen, oder dass die normale Entwicklung von Kindern durch solche beeinträchtigt wird.

- Die meisten mittels WGS generierten Daten lassen sich ohnehin nie klinisch nutzen. Sie haben eine zu geringe Bedeutung für die Gesundheit oder für die Therapierbarkeit einer Krankheit. Eine routinemässige Durchführung des WGS (Screening-Untersuchung) bei gesunden Individuen trennt die genetische Analytik von einer klinischen Indikation. Ohne klare klinische Fragestellung besteht die Gefahr einer Über-, aber auch einer Unterinterpretation der erhobenen Daten, da nicht fokussiert auf ein individuelles gesundheitliches Problem eingegangen wird. Zurückhaltung bei der Anpreisung/Vermarktung der WGS-Diagnostik ist daher angezeigt. Genetische Untersuchungen dürfen nicht für kommerzielle Zwecke und auch nicht für wissenschaftliche Ziele missbraucht werden. Die Zahl der vornehmlich genetisch bedingten Gesundheitsstörungen nimmt wegen des WGS ohnehin nicht zu. Man generiert damit keine neuen Erbkrankheiten.

- Der Wissenstand über Genetik ist in der schweizerischen Ärzteschaft sowie beim paramedizinischen Personal (inkl. der Pflegenden) meist recht bescheiden und reicht in der Regel für eine angemessene Beratung/Auskunft über molekulargenetisch generierte Daten nicht aus. Ratsuchende Laien können sich zudem leicht über Internet einen Informationsvorsprung aneignen. Daher besteht ein offensichtlicher Nachholbedarf in der Aus-, Weiter- und Fortbildung des gesamten Medizinalpersonals über moderne Genetik.

- Die dringend notwendige Zusammenarbeit für eine erfolgreiche Veranlassung und Durchführung genetischer Abklärungen sowie für die Interpretation ihrer Resultate ist zwischen Hausärzten, Spezialärzten, medizinischen Genetikern und Laborspezialisten in der Schweiz generell noch ungenügend eingespielt. Die vielerorts bereits etablierten Tumorboards könnten als Modell für künftige Genetikboards dienen. Zudem fehlt es an Experten, die genügend Wissen und Erfahrungen für eine bestmögliche Evaluation von WGS-Daten haben.

- Der Qualitätssicherung wird bei der Generierung genetischer Daten in unserem Lande grosses Gewicht beigemessen (siehe Verordnung über genetische Untersuchungen beim Menschen, GUMV). Eine solche ist nur in dafür zertifizierten Labors gestattet. Wie lässt sich aber die Qualitätssicherung bei der Vielfalt und Komplexität der beim WGS angewandten Methoden und der dafür benötigten Geräte im Labor und in der Informatik künftighin bewerkstelligen? Im Hinblick auf das WGS ist zudem eine internationale Zusammenarbeit sinnvoll. Universitäre Institutionen können davon profitieren, aber auch private Labors, die Zweigstellen im Ausland mit bereits mehr praktischen Erfahrungen im Umgang mit dem WGS haben. Untersuchungen würden dadurch bei uns rascher verfügbar, und dies vielleicht erst noch zu einem günstigeren Preis.

- Das Speichern und Übermitteln grosser Mengen von individuellen WGS-Daten ist technisch, aber auch rechtlich ungelöst. Die Informatik stellt eines der Hauptprobleme bei der Umsetzung des WGS für klinische Zwecke dar. Es bestehen grosse Sorgen im Hinblick auf eine nicht-autorisierte Nutzung von WGS-Daten.

- Die WGS-Technologie ist der Gesetzgebung unweigerlich weit voraus. Das Bundesgesetz über genetische Untersuchungen beim Menschen (GUMG) vom 8. Oktober 2004 basiert auf dem damaligen Wissen über die prä- und postnatale Diagnostik von Chromosomenaberrationen und Genmutationen mit hoher Durchschlagskraft (monogene Erbkrankheiten). Den dort geforderten Informed consent kann ein Proband wegen der Komplexität von WGS-Daten und der unvorher- 


\section{Translation genetischer Laborbefunde}

\section{Interpretation}

\section{Kommunikation}

$\left.\begin{array}{l}\text { Genetische } \\ \text { Labordaten } \\ \text { (Mutationen/Varianten) }\end{array} \quad \begin{array}{l}\text { Genetische } \\ \text { Informationen } \\ \text { (klinische Relevanz) }\end{array}\right) \begin{aligned} & \text { Individuelle } \\ & \text { ärztliche Hilfe }\end{aligned}$

\section{unter Berücksichtigung von:}

- Indikation

- Eigenschaften der Erbkrankheit
- Aussagekraft / Reichweite
Medizinisch-genetische Fakten plus:

- Psychosoziale Implikationen

- Ethische Aspekte

- Rechtliche Gegebenheiten
Die Nutzung von DNA-Sequenzierdaten im ärztlichen Alltag erfordert deren bestmögliche Interpretation, also Überführung in klinisch relevante Informationen nach wissenschaftlichen Kriterien, aber auch die einfühlsame Vermittlung der medizinisch-genetischen Erkenntnisse unter Berücksichtigung von psychischen, sozialen, ethischen und rechtlichen Gegebenheiten.

sehbaren Feststellung von DNA-Varianten mit möglicher klinischer Bedeutung nicht erteilen. Weitere Aspekte wollen geregelt sein: Wem gehören WGS-Daten, und wer darf sich ihrer bedienen? Eine gesetzliche Überregulierung des WGS könnte die vordringliche Forschung über die sinnvolle klinische Nutzung der Daten behindern und auch Ungerechtigkeiten schaffen. Nicht alle, die die neuen diagnostischen Möglichkeiten beanspruchen möchten, könnten dies auch tun, wenn sie dafür ins Ausland ausweichen müssten. Gesetze erweisen sich oft als zu starr, um mit dem raschen Fortschritt der Medizin Schritt halten zu können. Wir wissen heute noch nicht, was man in 10, 20 Jahren mit WGS-Daten alles anfangen kann. Statt einer staatlichen Bevormundung der Bevölkerung sowie der Ärzteschaft und statt einer Bürokratisierung der Inanspruchnahme von medizinischgenetischen Diagnostikverfahren mittels nur schwer kontrollierbaren Auflagen sind die Eigenverantwortung der Probanden und die Mitverantwortung der betreuenden Ärzteschaft zu fördern. Enge gesetzliche Vorschriften (eigentliche «Checklisten») dürfen die Letztere nicht von der menschlichen und intellektuellen Anteilnahme am individuellen Schicksal ihrer Patienten/Ratsuchenden entbinden.

- Die Erweiterung der genetischen Analytik im medizinischen Alltag vermehrt die Konfliktmöglichkeiten im Umgang mit genetischen Daten; diesbezüglich bereits genannt wurden der Datenschutz und die Besitzverhältnisse. Viele der von der Medizinethik für die medizinische Gene- tik entwickelten Prinzipien lassen sich schon im heutigen medizinischen Alltag kaum umsetzen. Wird eine Testperson mit dem auf dem Prinzip der Autonomie basierenden Selbstbestimmungsrecht nicht heute schon überfordert? Ein Informed consent zum WGS würde zu einer Zustimmung zu noch viel mehr Unbekanntem werden. Gleiches gilt auch für das Recht auf Wissen und Nicht-Wissen. Praktikable ethische Prinzipien sind zu entwickeln, deren Berücksichtigung den Respekt gegenüber der untersuchten Person und deren Schutz auch im WGS-Zeitalter gewährleisten kann.

- Eine angemessene Finanzierung der durch das WGS entstehenden Kosten ist eine Hauptvoraussetzung für dessen künftigen Einsatz im Praxisund Klinikalltag. Für Gentests herrscht diesbezüglich schon heute in der Schweiz Handlungsbedarf. Der nachfolgende Artikel von Felix Gutzwiller und Patricia Blank bringt deutlich zum Ausdruck, dass die Übernahme von Kosten für indizierte genetische Abklärungen durch die Krankenkassen nicht befriedigend gelöst ist. Die Forderung, dass die von diesen als Pflichtleistungen zu vergütenden Analysen nur für den Behandlungszweck erforderlich sein müssen, ist nicht vertretbar. Das Bundesgesetz über die Krankenversicherung (BVG) bedarf diesbezüglich dringend der Revision. Präventivmassnahmen, basierend auf dem Nachweis von krankheitsverursachenden Veranlagungen, gewinnen an praktischer Bedeutung. Es liegt zudem gerade im Interesse vieler Patenten, zu wissen, ob ihre Krankheit wegen einer molekulargenetisch nachweisbaren Veranlagung entstanden ist oder nicht. Sie machen sich berechtigte Sorgen um die Gesundheit ihrer Kinder und wünschen daher einen Gentest, damit diesen allenfalls eine verlässliche präsymptomatische Diagnostik und falls indiziert - angemessene Vorsorgeuntersuchungen angeboten werden können. Gerade für zahlreiche «hereditäre» Tumorkrankheiten ist zudem gut dokumentiert, dass regelmässige Vorsorgeuntersuchungen und frühzeitige therapeutische Massnahmen viel kostengünstiger sind als die oft wenig aussichtsreiche Behandlung einer fortgeschrittenen Krebskrankheit.

«The future of genomic sequencing is very bright!» Dies hört man besonders oft von Stimmen aus den USA. Die Aussage kann zutreffen, falls es gelingen wird, einen verantwortungsvollen Umgang mit dieser Teststrategie und den daraus hervorgehenden Daten zu schaffen. Bei all den Erwartungen in eine künftige Medizin mit WGS darf man Altbewährtes jedoch nicht vergessen und vernachlässigen: so die krankheitsbezogene Familienanamnese als ein kostengünstiges und sehr informatives genetisches Diagnoseverfahren sowie die Bedeutung der einfühlsamen, verantwortungsvollen, sehr persönlichen Arzt-Patienten-Beziehung. 